\title{
Measurement of Plasma Potential, Ion Saturation Current and Mach number using Retarding Field Energy Analyzer
}

\section{N. Mishra and A. Fredriksen}

Journal of Nepal Physical Society

Volume 7, Issue 2, June 2021

ISSN: 2392-473X (Print), 2738-9537 (Online)

Editors:

Dr. Binod Adhikari

Dr. Bhawani Joshi

Dr. Manoj Kumar Yadav

Dr. Krishna Rai

Dr. Rajendra Prasad Adhikari

Mr. Kiran Pudasainee

JNPS, 7 (2), 76-80 (2021)

DOI: https://doi.org/10.3126/jnphyssoc.v7i2.38626

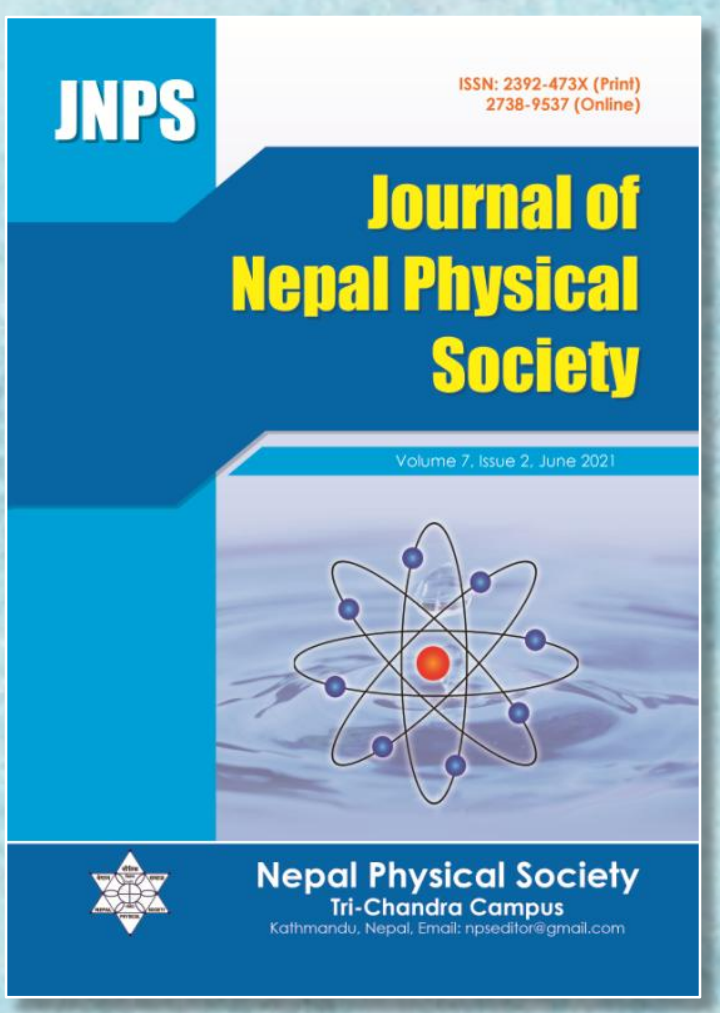

Published by:

Nepal Physical Society

P.O. Box: 2934

Tri-Chandra Campus

Kathmandu, Nepal

Email: nps.editor@gmail.com 


\title{
Measurement of Plasma Potential, Ion Saturation Current and Mach number using Retarding Field Energy Analyzer
}

\author{
L. N. Mishra ${ }^{1}$ and Å. Fredriksen ${ }^{2}$ \\ ${ }^{1}$ Patan Multiple Camus, Tribhuvan University, Patan, Lalitpur, Nepal \\ ${ }^{2}$ Department of Physics, Faculty of Science, University of Tromso, Norway \\ Corresponding Email: lekha.mishra@pmc.tu.edu.np
}

Received: 3 April, 2021; Revised: 13 May, 2021; Accepted: 19 June, 2021

\begin{abstract}
This article deals about the experimental measurement of plasma potential, ion saturation current and Mach number obtained with the variation of power, operating gas pressure and radial position using retarding field energy analyzer. We employed a retarding field energy analyzer by rotating with different angles such as $0^{\circ}$ (facing toward source), $90^{\circ}$ (facing side walls) and $180^{\circ}$ (facing opposite the source). The coil current is varied from 0 to $15 \mathrm{~A}$ to produce the magnetic field which is used to confine the plasma. The flow of plasma has been characterized which was found to be subsonic. The low-temperature plasma is produced by means of a $13.56 \mathrm{MHz}$ helicon plasma source at $300-1000 \mathrm{~kW}$ radio frequency power. The plasma is expanding from $13.8 \mathrm{~cm}$ diameter source into a $150 \mathrm{~cm}$ long diffusion chamber of 60 cm diameter.
\end{abstract}

Keywords: Plasma flows, Helicon plasma, Space plasma.

\section{INTRODUCTION}

Surface modification technique is a most powerful tool using in modern industry to fabricate the electronic devices [1-6]. In this process, substrates are exposed to plasma for several hours where they are bombarded by energetic ions. Due to flux of bombarding energetic ions, removal and deposition [7-10] of layers takes place on the substrate. The former process is called etching where radio frequency (rf) inductively coupled plasma [11-14] reactor is used. To diagnose such a plasma, retarding field energy analyzer (RFEA) is employed to measure the plasma potential, ion saturation current, electron temperature, electron density, ion density, Mach number and so on. It is well-known that the flow of ions and electrons play the crucial role in space plasma as well as plasma processing to the surface modifications of materials for a wide variety of industrial applications. In the former case, ions collide much more frequently with the neutral molecules than with other ions at auroral latitudes. These phenomena could be carried out by taking the information on the distribution either from high resolution ground- based optical measurements or from retarding potential analyzers carried on rockets and satellites. But, in later case, the microelectronic industry use plasma discharges for the processing of semiconductor wafers. So, the mechanism or dynamics of ions or electrons interact to the semiconductors surface due to their bombardment is quite important where they interchange their energies.

However, the flux of positive ions and its energy determine the effectiveness of plasma process. More specifically, it is more challenges to researchers seeking to measure such properties in the laboratory because plasmas are hot, and at sufficiently high densities, they can deposit enough heat to vaporize any material put into or around them, including containment vessels or probes. Additionally, since plasmas are locally charged, they interact strongly with electromagnetic waves. This property makes the probing of the interior behavior of plasmas complicated, although it also offers unusual diagnostic opportunities. Also, any electrical measurements of the plasma tend to perturb the plasma in some way, which can 
complicate any effort to learn about the unperturbed state of the plasma [15]. In this paper, we present the measurement plasma potential, ion saturation current and Mach number obtained by RFEA in the downstream region of $\mathrm{rf}$ source. Measurements were carried out in different guiding coil currents.

\section{EXPERIMENTAL SET UP}

(a)

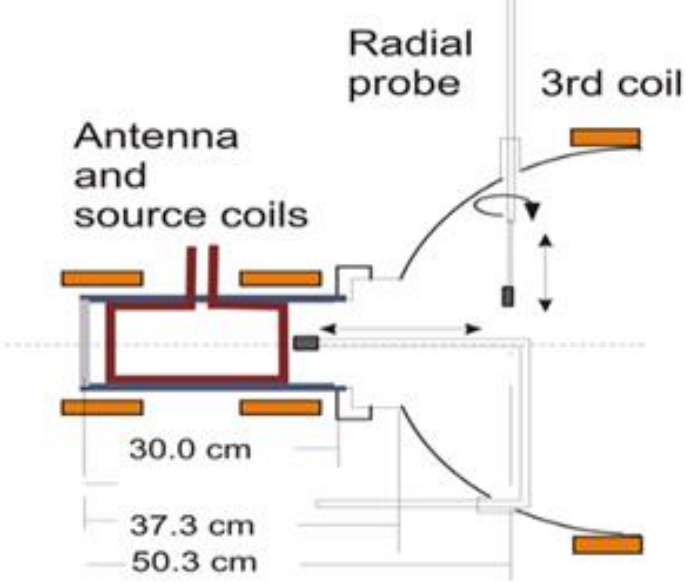

(b)

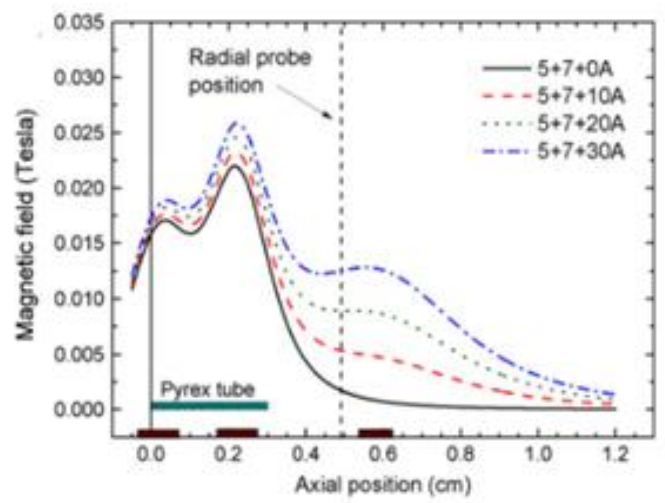

Fig. 1: Experimental arrangement: RF source with probe (a) and magnetic field magnetic field configuration (b).

The experimental arrangement for the measurement of plasma potential, ion saturation current and Mach number of argons rf plasma in the Njord device is shown as in Fig. 1. It consists of a source $(\phi 21 \mathrm{~cm} \times 31.3 \mathrm{~cm})$ attached to a vacuum chamber made of stainless steel, called the diffusion chamber $(\phi 60 \mathrm{~cm} \times 120 \mathrm{~cm})$. More specifically, plasma is formed in a Pyrex tube $(\phi 13.8 \mathrm{~cm} \times 30.5$ $\mathrm{cm})$ called the source chamber. The source and diffusion chambers are connected at a cylindrical port having dimension $\phi 20 \mathrm{~cm} \times 6 \mathrm{~cm}$. In the present work, we have adopted the plasma source which was similar to "Chi-Kung" at Australian National University [16]. It consists of a double saddle rf-antenna fed with $13.56 \mathrm{MHz}$ from a Henry $8 \mathrm{~K}$ Ultra rf amplifier and is fitted with a pinetwork tuning system with vacuum capacitors. The rf power applied for the breakdown of the gas is in the range $200-600 \mathrm{~W}$.

A weak magnetic field of maximum $25 \mathrm{mT}$ is applied in the source region by means of two coils outside the saddle-type helicon antenna placed around the Pyrex tube source chamber. In addition, third coil called guiding coil is placed $58 \mathrm{~cm}$ downstream from the end plate of the source, which defines the origo along the axis of the source. The field produced by the third coil could be varied from 0 to $15 \mathrm{~A}$, so that an inflection point is

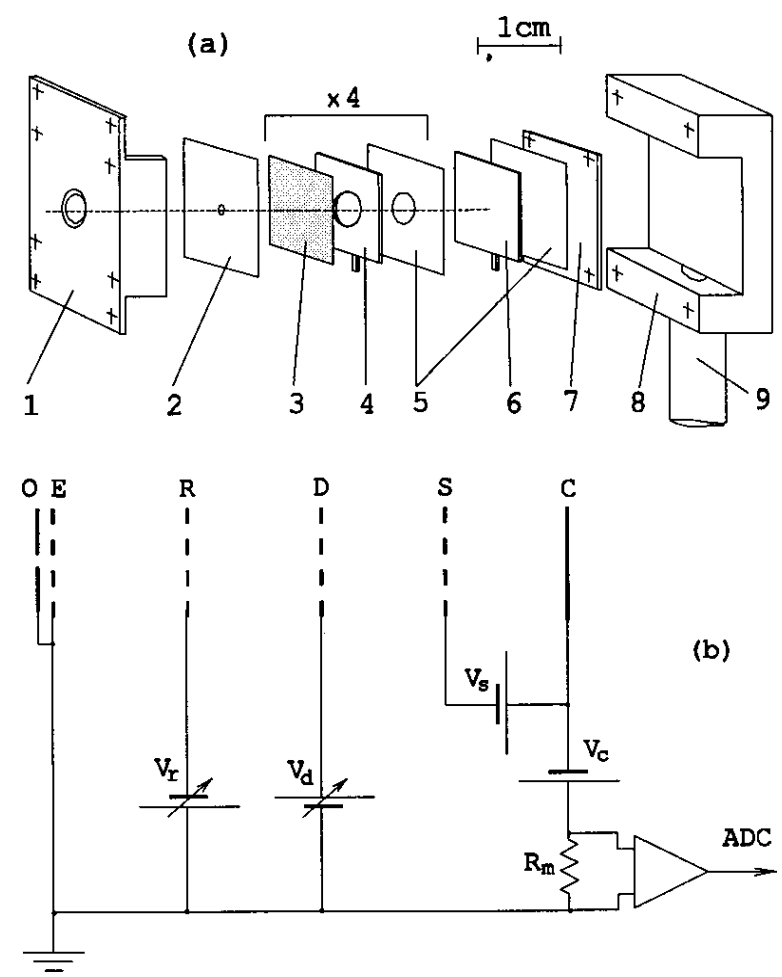

Fig. 2: RFEA design (a) and biasing (b). 1-analyzer lid, 2-orifice plate, 3-steel mesh, 4-copper support plate, 5-mica insulators, 6-collector, 7-clamp plate, 8-analyzer body, 9-support tube, $\mathrm{O}$-orifice plate, $E$-entrance grid, $R$-repeller grid, $D$-discriminator grid, $S$-suppressor grid, $C$-collector. 
produced between the second and third coil, as indicated in fig. 1(a) and 1(b). The vacuum system in the working chambers consist of one turbomolecular pump backed by one $350 \mathrm{l} / \mathrm{min}$ rotary pump, with which the base pressure of the system was kept below $2.67 \times 10^{-8}$ bar. Its operating pressure is $\leq 2.39 \times 10^{-7}$ bar. The flow control is carried out by a Omega FMA 5400/5500 series flow controller with maximum capacity 20 $\mathrm{cm}^{3} \mathrm{~min}^{-1}$. This controller is driven with remote computer system.

A retarding field energy analyzer (RFEA) shown in Fig. 2 was inserted in a radial direction of source chamber where plasma produced in this chamber flows to the diffusion chamber keeping low gas pressure typically at $2.39 \times 10^{-7}$ bar. The latter RFEA could be rotated thoroughly $360^{\circ}$ around its axis. This RFEA had orifice diameter $1 \mathrm{~mm}$, and was constructed with a mesh across the orifice. Its electron repeller grid was biased at $-90 \mathrm{~V}$, the discriminator grid had variable bias from $-100 \mathrm{~V}$ to $+100 \mathrm{~V}$, the secondary repeller grid was biased at $20 \mathrm{~V}$ and the collector at $-9 \mathrm{~V}$. The overall system length was $2 \mathrm{~mm}$ with $0.50 \mathrm{~mm}$ space between the grids. There is good electrical connection between the plate and the grounded analyzer housing. The discriminator was biased in 500 steps per scan from $-100 \mathrm{~V}$ to $+100 \mathrm{~V}$. At each step the collector current, measured over a $32 \mathrm{k} \Omega$ resistor, was digitized into 500 samples which were then averaged into one single value, and written to file for further analysis. Figure 2 is a RFEA bias circuit: E - earthed, R repeller, $\mathrm{D}$ - discriminator, $\mathrm{S}$ - secondary emission suppressor, C - collector.

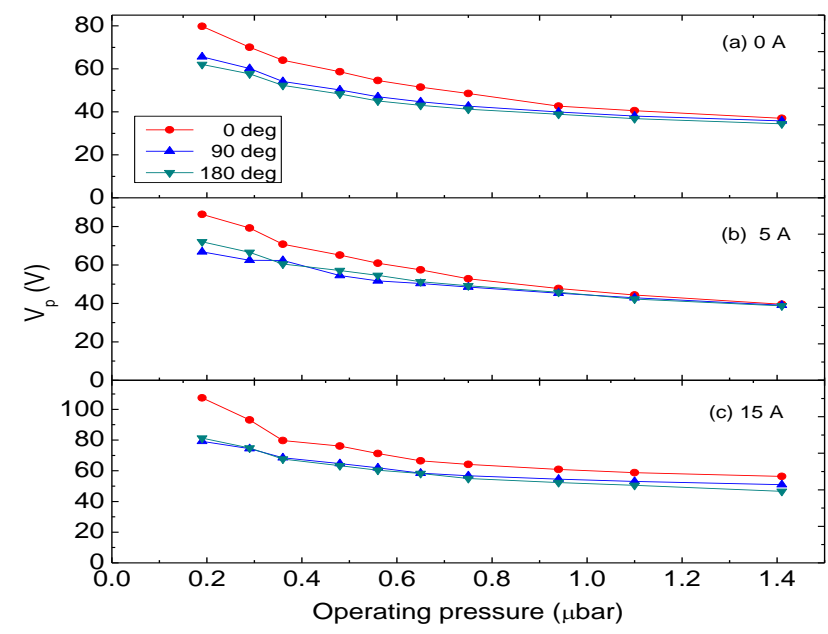

Fig. 3: The variation of plasma potentials as function of operating gas pressure.

\section{RESULTS AND DISCUSSION}

The key parameters of background rf plasma are expressed in terms of plasma potential. In this case, we employed a retarding field energy analyzer by rotating with different angles such as $0^{0}$ (facing toward source), $90^{\circ}$ (facing side walls) and $180^{\circ}$ (facing opposite the source) to measure the plasma potential. Figure 3 shows the variation of plasma potentials as function of operating gas pressure for three guiding coil currents and three above mentioned rotating angles.

It is found that plasma potential decreases as the gas pressure increases. It infers from the Fig. 3 that at the zero or low magnetic field maintaining $0 \mathrm{~A}$ i.e. no current providing in third coil, a large number of plasma particles diffuse to the walls, which enhances the reduction of background plasma. But, when the magnetic field increases by changing the current from 5 to $15 \mathrm{~A}$, all the fluxes are staying between source chamber and the neck of diffusion chamber which enhances the increment of plasma potential.

In the presence of a magnetic field, the gyration of electrons and ions are opposite to each other. Hence, transport of electrons and ions is altered and thus the properties of the plasma are affected.

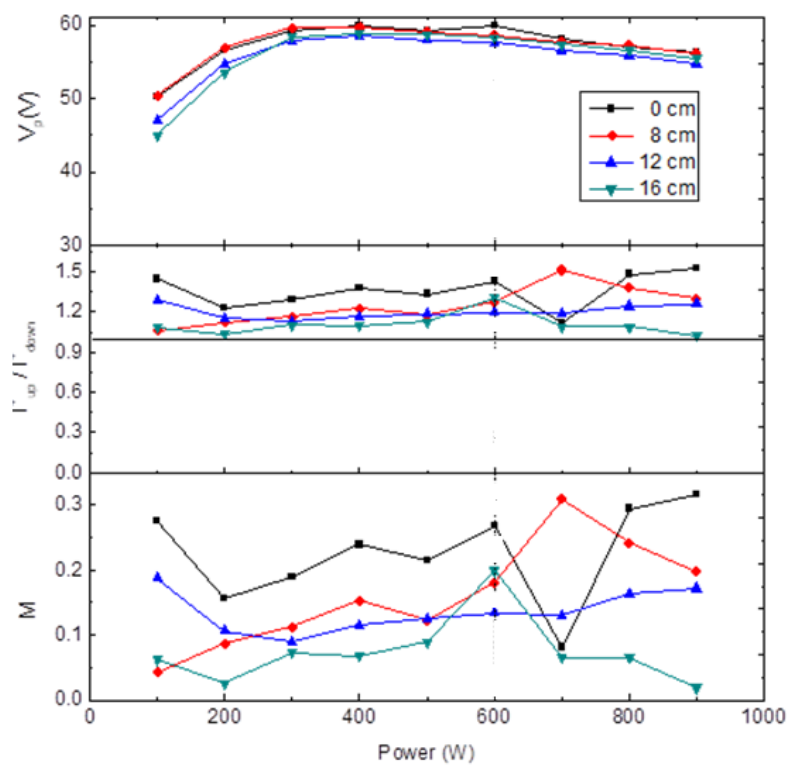

Fig.4: A typical profile of plasma potential, ratio of ion saturation current and Mach number as the function of input power.

Figure 4 is the typical profile of plasma potential, ratio of ion saturation current and Mach number as the function of input power. It is revealed that the upstream ion saturation current is larger than that of 
downstream ion saturation currents which infers that the flow of ions from upstream to downstream direction. Actually, it does not give the actual flow measurements because the RFEA itself a large grounded object which creates a wake. The Mach number can be measured by the formula

$M=\frac{1}{K} \ln \left(\frac{I_{u p}}{I_{\text {down }}}\right)=\frac{v_{f l}}{c_{s}} \quad$ [17] where $\mathrm{K}=1.45$ for magnetized plasma and 1.26 for non-magnetized plasma. Higher the Mach number more the flow velocity.

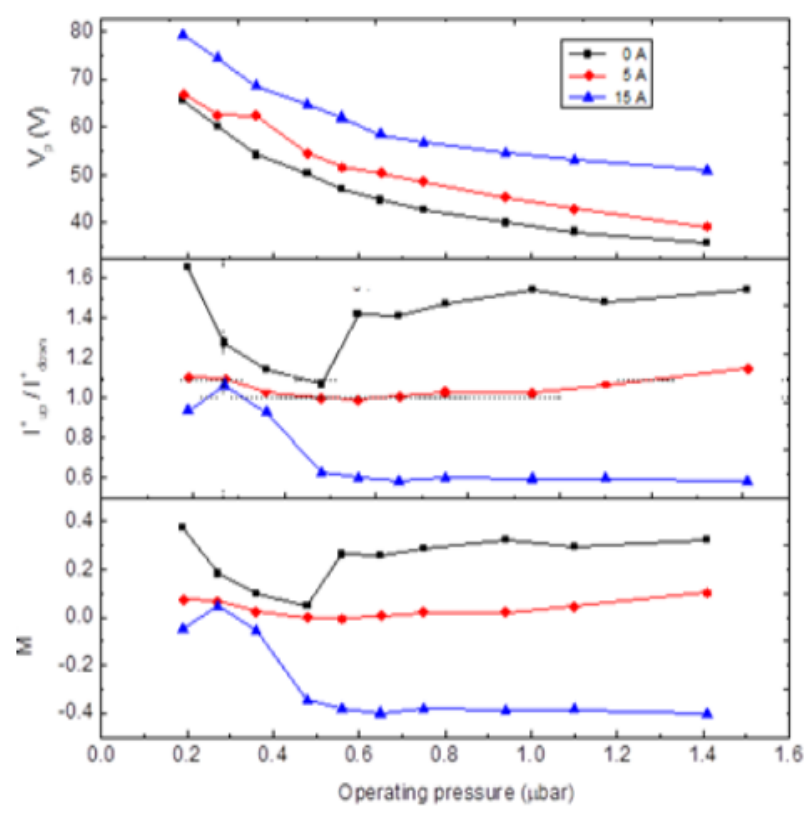

Fig. 5: A typical profile of plasma potential, ratio of ion saturation current and Mach number as the function of operating pressure.

Figure 5 is a typical profile of plasma potential, ratio of ion saturation current and Mach number as the function of operating pressure. In this case, third coil current is taken as $0 \mathrm{~A}, 5 \mathrm{~A}$ and $15 \mathrm{~A}$. It is found that plasma potential decreases as operating pressure increases whereas ion saturation current ratio and Mach number fluctuates. In our case, Mach number is found to be less than 0.4. This shows that flow is subsonic because subsonic condition occurs when Mach number is less than 1 .

\section{CONCLUSIONS}

The experimental measurement of plasma potential, ion saturation current and Mach number obtained with the variation of power, operating gas pressure and radial position using retarding field energy analyzer have been elucidated. These plasma parameters are also studied in the configuration of different magnetic field by providing the different current in guiding coil. The coil current can be varied from 0 to $15 \mathrm{~A}$ to produce the magnetic field which is used to confine the plasma. A retarding field energy analyzer was employed by rotating with different angles such as $0^{0}$ (facing toward source), $90^{\circ}$ (facing side walls) and $180^{\circ}$ (facing opposite the source). The flow of plasma has been characterized which was found to be subsonic where Mach number is found to be less than 0.4. It is found that plasma potential increases as guiding coil increases in third coil. In the presence of a magnetic field, the gyration of electrons and ions are opposite to each other. Hence, transport of electrons and ions is altered and thus the properties of the plasma are affected.

\section{ACKNOWLEDGEMENTS}

This work was supported by Norwegian Research Council, Grant no. 177570.

\section{REFERENCES}

1. Mozetic, M. Surface modification to improve properties of materials. Materials, 12: 441 (2019).

2. Abou Rich, S.; Dufour, T.; Leroy, P.; Reniers, F.; Nittler, L. \& Pireaux, J.-J. LDPE surface modifications induced by atmospheric plasma torches with linear and showerhead configurations. Plasma Process. Polym., 12: 771785 (2015).

3. Esbah Tabaei, P.S.; Ghobeira, R.; Cools, P.; Rezaei, F.; Nikiforov, A.; Morent, R. \& De Geyter, N. Comparative study between in-plasma and post-plasma chemical processes occurring at the surface of UHMWPE subjected to medium pressure Ar and N2 plasma activation. Polymer, 193: 122383 (2020).

4. Pandiyaraj, K.N.; Deshmukh, R.R.; Ruzybayev, I.; Shah, S.I.; Su, P.-G.; Halleluyah, J.M. \& Halim, A.S. Influence of non-thermal plasma forming gases on improvement of surface properties of low density polyethylene (LDPE). Appl. Surf. Sci., 307: 109-119 (2014).

5. Bílek, F.; Krížová, T. \& Lehocký, M. Preparation of active antibacterial LDPE surface through multistep physicochemical approach: I. Allylamine grafting, attachment of antibacterial agent and antibacterial activity assessment. Colloids Surf. B, 88: 440-447 (2011).

6. Jacobs, T.; Morent, R.; De Geyter, N.; Dubruel, P. \& Leys, C. Plasma surface modification of biomedical polymers: Influence on cell-material interaction. Plasma Chem. Plasma P, 32: 10391073 (2012). 
7. Mishra, L. N.; Shibata, K.; Ito H., Yugami, N. \& Nishida, Y. Characteristics of electron Cyclotron resonance plasma Generated in a rectangular wave guide by high power Microwave. Review of Scientific Instruments, 75: 84-89 (2004).

8. $\quad$ Mishra, L. N.; Shibata, K.; Ito H., Yugami, N. \& Nishida, Y. Pulsed Corona Discharge as a Source of Hydrogen and Carbon Nanotube Production, IEEE Transaction on Plasma Science, 32: 17271733 (2004).

9. Quitzau, M.; Wolter, M. \& Kersten, H. Plasma treatment of polyethylene powder particles in a hollow cathode glow discharge. Plasma Process. Polym., 6: 392-396 (2009).

10. Tkavc, T.; Petrinič, I.; Luxbacher, T.; Vesel, A.; Ristić, T. \& Zemljič, L.F. Influence of $\mathrm{O} 2$ and $\mathrm{CO} 2$ plasma treatment on the deposition of chitosan onto polyethyleneterephthalate (PET) surfaces. Int. J. Adhes Adhes, 48: 168-176 (2014).

11. Fredriksen, Å.; Mishra L. N. \& Byhring H.S. The effects of downstream magnetic field on currentfree double layers and beam formation in the Njord helicon plasma device, Plasma Sources Sci. Technol, 19: 5 (2010).

12. Miloch W.J; Gulbrandsen N.; Mishra L. N. \& Fredriksen $\AA$. Ion velocity distribution in the sheath and presheath of a biased object in plasma, Physics of Plasmas, 18: 083502 (2011).

13. Fredriksen, Å; Mishra L. N.; Gulbrandsen N. \& Miloch W. J. On the Measurement of Subsonic Flow in a Capacitively Coupled Helicon Plasma Source, J. Phys. Conf. Ser., 257: 012019 (2010).

14. Eckert H. U. AIAA paper no. 68-711, presented at the AIAA Fluid and Plasma Dynamics Conference, Los Angeles, CA, June 24-26, (1968).

15. Tsaur, B. Y.Thin Film Interfaces and Interactions, Baglin, J. E. E., Poate, J. M. eds. (The Electrochemical Society, Princeton 1980), Proceedings, 80(2): 205 (1980).

16. Charles, C.; Boswell, R. W. \& Porteous, R. K. Current-free double-layer formation in a highdensity helicon discharge, Appl. Phys. Lett., 82: 1356 (2003).

17. Chung, K.S. \& Hutchinson, I.H. Plasma flow measurements along the presheath of a magnetized plasma, Phys. Fluids B1, 2229 (1989). 